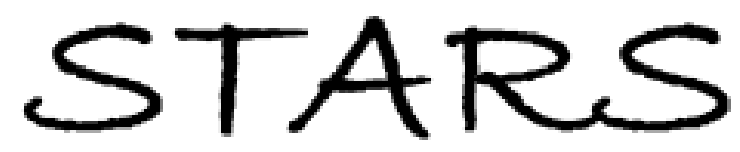

University of Central Florida

STARS

$1-1-2013$

\title{
Subwavelength light confinement and propagation: A numerical study of a two-layer silver film with perforated holes
}

Jennifer M. Reed

University of Central Florida

Haining Wang

University of Central Florida

Yingnan Guo

University of Central Florida

Shengli Zou

University of Central Florida

Find similar works at: https://stars.library.ucf.edu/facultybib2010

University of Central Florida Libraries http://library.ucf.edu

This Article is brought to you for free and open access by the Faculty Bibliography at STARS. It has been accepted for inclusion in Faculty Bibliography 2010 s by an authorized administrator of STARS. For more information, please contactSTARS@ucf.edu.

\section{Recommended Citation}

Reed, Jennifer M.; Wang, Haining; Guo, Yingnan; and Zou, Shengli, "Subwavelength light confinement and propagation: A numerical study of a two-layer silver film with perforated holes" (2013). Faculty Bibliography 2010s. 4583.

https://stars.library.ucf.edu/facultybib2010/4583

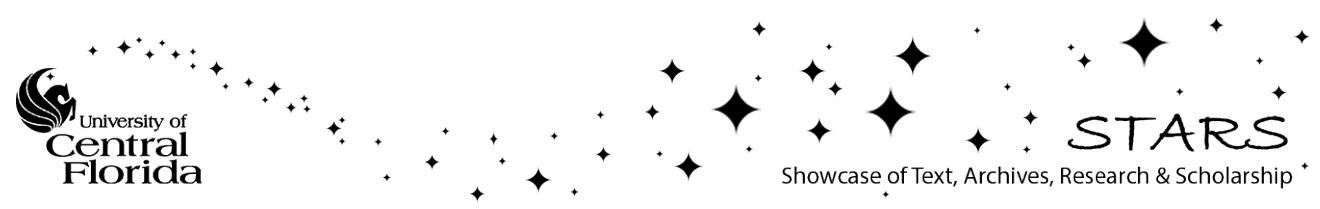




\section{Subwavelength light confinement and propagation: A numerical study of a two- layer silver film with perforated holes}

Cite as: Appl. Phys. Lett. 103, 161114 (2013); https://doi.org/10.1063/1.4826441

Submitted: 15 August 2013 . Accepted: 07 October 2013 . Published Online: 17 October 2013

Jennifer M. Reed, Haining Wang, Yingnan Guo, and Shengli Zou
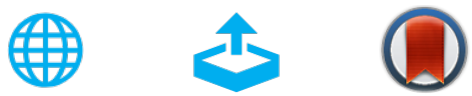

\section{ARTICLES YOU MAY BE INTERESTED IN}

Universal formalism of Fano resonance

AIP Advances 5, 017137 (2015); https://doi.org/10.1063/1.4906797

An analytic model for the optical properties of gold

The Journal of Chemical Physics 125, 164705 (2006); https://doi.org/10.1063/1.2360270

A comprehensive review of $\mathrm{ZnO}$ materials and devices

Journal of Applied Physics 98, 041301 (2005); https://doi.org/10.1063/1.1992666

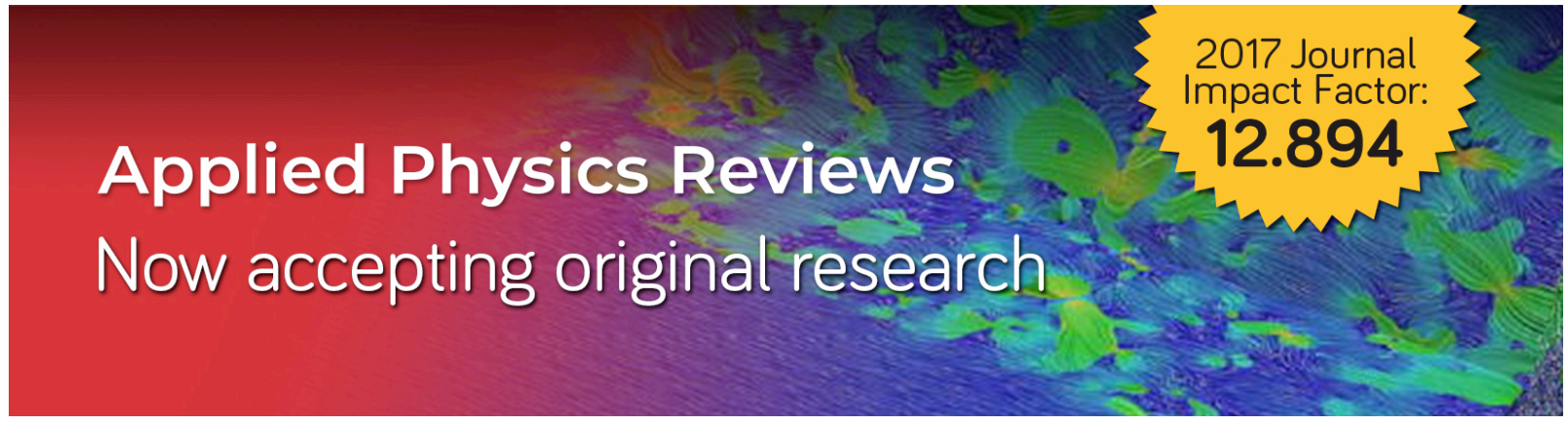




\title{
Subwavelength light confinement and propagation: A numerical study of a two-layer silver film with perforated holes
}

\author{
Jennifer M. Reed, ${ }^{1}$ Haining Wang,,${ }^{1,2}$ Yingnan Guo, ${ }^{1,3}$ and Shengli Zou ${ }^{1, a)}$ \\ ${ }^{1}$ Department of Chemistry, University of Central Florida, 4104 Libra Drive, Orlando, Florida 32816-2366, USA \\ ${ }^{2}$ Beijing Key Laboratory of Bio-inspired Energy Materials and Devices, School of Chemistry and Environment, \\ Beihang University, Beijing 100191, China \\ ${ }^{3}$ School of Physics and Optoelectronic Technology, Dalian University of Technology, Dalian 116024, China
}

(Received 15 August 2013; accepted 7 October 2013; published online 17 October 2013)

\begin{abstract}
Confinement and propagation of light under the diffraction limit is a substantial challenge for the development of waveguides as components in subwavelength optical devices. Light confinement and propagation at optical frequencies in a two-layer silver film with a periodic array of holes is investigated in a numerical study using the discrete dipole approximation method. Extraordinary subwavelength confinement and propagation of light can be obtained along an extremely subwavelength cross section of a region as small as $10 \times 20 \mathrm{~nm}^{2}$ at a distance of over $10 \mu \mathrm{m}$. This study provides rational design of waveguides under subwavelength dimensions. (C) 2013 AIP Publishing LLC. [http://dx.doi.org/10.1063/1.4826441]
\end{abstract}

Advancement in modern electronics is characterized by the continual decreasing of device size and increasing of device processing speed. However, it is limited by the speed of electron transport in the components of the device. ${ }^{1}$ The utilization of electromagnetic waves, instead of electrons, as information carriers circumvents this problem. In a photonic device, electromagnetic waves travel via waveguides that confine and guide wave propagation, which is analogous to conductive wires in an electronic device channeling electrons. Still, with the use of optical information carriers in nanoscale waveguides, a new challenge arises: localizing and propagating the electromagnetic waves beyond the diffraction limit. Yet, at optical wavelengths, metals such as gold and silver exhibit a collective oscillation of conduction electrons, called surface plasmons (SPs). Metal interfaces can support evanescent surface waves called surface plasmon polaritons (SPPs). Since the SPP wavelength is smaller than the wavelength of light in a vacuum, the diffraction limit can be diminished in device design. Various methods have shown that the size of the waveguide and other nanostructured devices can be reduced beyond the diffraction limit of light using surface plasmons. ${ }^{2-7}$ Furthermore, integrating of nanoscale waveguide components into a functional system has shown that the realization of subwavelength photonic devices is attainable. ${ }^{8}$

In this Letter, we propose a subwavelength photonic waveguide, which consists of a two-layer, metal-insulatormetal (MIM) film structure with perforated holes to meet three main goals of waveguide design: (1) trapping, (2) confining, and (3) propagating light. From our previous work, where we focused on the first goal of waveguide design using conical frustum shaped holes in a two layer silver film, we concluded that a 1 to 3 critical ratio of the upper and lower diameters of the holes was necessary to achieve high absorption due to the reduced reflection of incident light perpendicular to the layers. ${ }^{9,10}$ In the current work, we focus on

\footnotetext{
${ }^{\text {a) }}$ Author to whom correspondence should be addressed. Electronic mail: shengli.zou@ucf.edu.
}

confining and propagating the light; therefore, the upper and lower diameters of the holes are kept the same so as to take advantage of the light trapping properties without sacrificing other waveguide design goals. We demonstrate that the light propagation is related to the electric field confinement in the narrow region between the two layers and that the light from a single hole can propagate in an enclosed channel. The structure can be tuned so that propagation can be achieved for a variety of wavelengths, regardless of the resonance frequency, making it more versatile than resonance dependent structures. We calculate out the effective index theoretically so as to explain such phenomenon, which agrees well with our simulations. The results have potential applications for subwavelength waveguide design.

Using the Discrete Dipole Approximation (DDA) method, we begin by first investigating the effects of varying different structural components of the waveguide: the hole diameter and the distance between the two silver layers. The DDA method, which was first proposed by Purcell and Pennypacker ${ }^{11}$ and developed by Draine, ${ }^{12}$ is a commonly used program which calculates the optical properties of films or particles with arbitrary shapes and dielectric properties of the materials. The dielectric constants of silver are taken from Palik's handbook. ${ }^{13}$ In the DDA method, the target is divided into $\mathrm{N}$ small cubes, which are considered as polarizable dipoles. After solving 3N linear equations, the induced dipole and local electric field of each cube are obtained, followed by the calculation of the amplitude scattering matrix, which relates the incident and scattered light. The Mueller matrix, which relates the incident and scattered waves, is calculated from the amplitude scattering matrix and gives the scattering and transmission spectra of the target. ${ }^{14}$

To begin investigating the light trapping, we vary the distance between the films, L, as shown in Fig. 1, and the hole diameter, d, with holes periodic every $400 \mathrm{~nm}$ along the $\mathrm{Y}$ axis and every $10 \mu \mathrm{m}$ along the $\mathrm{Z}$ axis. The thickness of the silver layers, $t_{1}$ and $t_{2}$, is fixed at $100 \mathrm{~nm}$ so that the layers are optically thick enough to prevent transmission. The incident light propagates along the $\mathrm{X}$ axis and the 

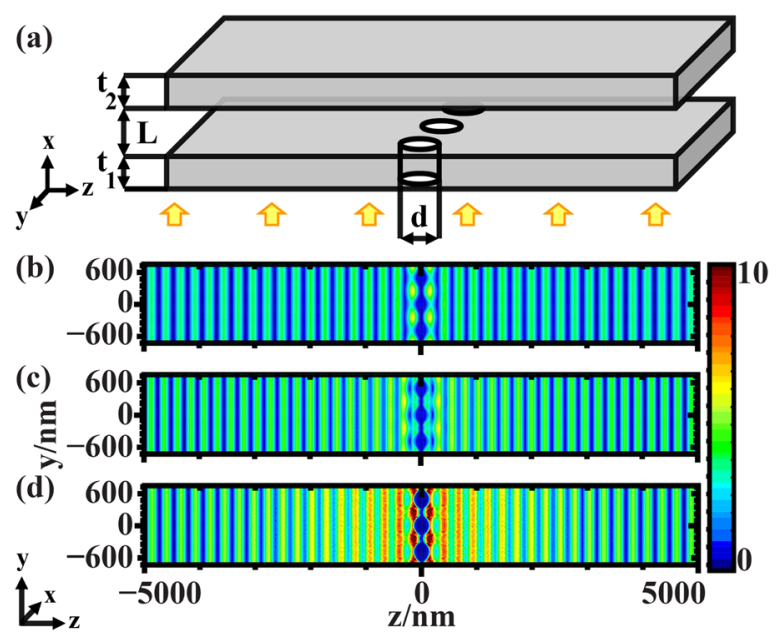

FIG. 1. (a) Schematic of an open array of holes periodic at $10 \mu \mathrm{m}$ along the $\mathrm{Z}$ axis and $400 \mathrm{~nm}$ along the $\mathrm{Y}$ axis. Electric field contour plots between the two layers in the YZ plane with a distance between films of (b) $40 \mathrm{~nm}$ and (c) $30 \mathrm{~nm}$ at a wavelength of $800 \mathrm{~nm}$ and (d) $10 \mathrm{~nm}$ at a wavelength of $1300 \mathrm{~nm}$.

polarization is along the $\mathrm{Z}$ axis. Initial electric field contour plots were calculated for hole diameters of 200, 300, and $400 \mathrm{~nm}$ for film distances of 50, 40, 30, 20, and $10 \mathrm{~nm}$. We found that holes with $300 \mathrm{~nm}$ diameters showed the highest efficiency for light trapping between the two layers. For the purpose of this paper, only the optimized results from the diameter tests of $300 \mathrm{~nm}$ are shown.

Figs. 1(b) and 1(c) show the electric field contour plots, $|\mathrm{El}|^{2}$, for Z polarized incident light at a wavelength of $800 \mathrm{~nm}$ with varied interfilm distance, L, and fixed diameter, $\mathrm{d}=300 \mathrm{~nm}$. Efficient light propagation is observed as alternating bright and dark fringes, which result from the interference between the holes along the $\mathrm{Z}$ axis, where the distance between bright fringes is half of the wavelength of the SPP mode excited at the interface of the top and bottom metal films. For a single metal-dielectric interface, the wavelength of the SPP mode, $\lambda_{\mathrm{spp}}=2 \pi / \mathrm{k}_{\mathrm{spp}}$, where $\mathrm{k}_{\mathrm{spp}}$ is the wavevector of the SPP mode and is related to the dielectric constant of the dielectric material, $\varepsilon_{\mathrm{d}}$, through the surface plasmon dispersion relation

$$
k_{s p p}=k_{0} \sqrt{\frac{\varepsilon_{m} \varepsilon_{d}}{\varepsilon_{m}+\varepsilon_{d}}},
$$

where $\mathrm{k}_{0}=2 \pi / \lambda_{0}$ is the free space wavevector with a wavelength of $\lambda_{0}$, and $\varepsilon_{\mathrm{m}}$ is the dielectric constant of the metal. The dispersion relation gives the factor needed to increase the momentum of a free space photon, $\hbar k_{0}$, to match that of an excited SPP mode wavevector $\hbar k_{\text {spp }}$ at a metal-dielectric interface. However, due to the presence of the second film, $\varepsilon_{\mathrm{d}}$ inside the waveguide is increased resulting in a new "effective" dielectric constant, $\varepsilon_{\text {d,eff }}$. Therefore, $\lambda_{\text {spp }}$ in a MIM system is what we refer to as the "effective" wavelength, and $\varepsilon_{\mathrm{d}}$ in Eq. (1) becomes $\varepsilon_{\mathrm{d} \text {,eff. }}$.

To confine the light and demonstrate the dependency of the SPP mode on $\varepsilon_{\mathrm{d} \text {,eff }}$, L is decreased from $40 \mathrm{~nm}$ to $30 \mathrm{~nm}$ at a fixed $\lambda_{0}=800 \mathrm{~nm}$, the intensity of the electric field between the two layers increases while at the same time maintaining efficient light propagation, as shown in Figs. 1(b) and 1(c), respectively. In conjunction with the increased intensity, a decreased $\lambda_{\text {spp }}$ is observed from $\lambda_{\text {spp }}=556 \mathrm{~nm}$ for $\mathrm{L}=40 \mathrm{~nm}$ to $\lambda_{\mathrm{spp}}=500 \mathrm{~nm}$ for $\mathrm{L}=30 \mathrm{~nm}$. L is decreased to an incredibly small channel height of $10 \mathrm{~nm}$ at $\lambda_{0}=1300 \mathrm{~nm}$ to demonstrate that efficient light propagation with different distances between films, while preserving the SPP wavelength, is still achievable, as shown in Fig. 1(d). The resulting SPP wavelength, for $\mathrm{L}=10 \mathrm{~nm}$, is $\lambda_{\mathrm{spp}}=556 \mathrm{~nm}$ for $\lambda_{0}=1300 \mathrm{~nm}$, which is the same as that of the $\mathrm{L}=40 \mathrm{~nm}$ structure with $\lambda_{0}=800 \mathrm{~nm}$. Therefore, tuning of the waveguide device can be done by decreasing the film spacing to decrease the resulting SPP wavelength and the propagation efficiency of the waveguide has little dependency on the resonance frequency of the structure.

The change of the effective wavelength versus the film distance can be explained by replacing $\varepsilon_{\mathrm{d}}$ in Eq. (1), with $\varepsilon_{\mathrm{d} \text {,eff }}$, and thus returning the "effective" wavelength in the waveguide. From Figs. 1(b)-1(d), it is seen that decreasing L increases $\mathrm{k}_{\mathrm{spp}}$, which means there must be an increase in the effective refractive index, $\mathrm{n}_{\mathrm{eff}}=\sqrt{\varepsilon_{d}}$, in the channel to satisfy the momentum matching criteria of the dispersion relation. Neglecting the effects of the holes in the film and treating the system as a MIM layer structure, $n_{\text {eff }}$ can be solely approximated from $\varepsilon_{\mathrm{d}}, \varepsilon_{\mathrm{m}}$, and $\mathrm{L}$, through the free space wavevector, $\mathrm{k}_{0}$, and propagation constant, $\beta$ (Ref. 15)

$$
n_{e f f}^{2}=\left(\frac{\beta}{k_{0}}\right)^{2}=\varepsilon_{d, e f f},
$$

where $\beta$ comes from

$$
-\frac{\varepsilon_{\mathrm{d}} \sqrt{\beta^{2}-\mathrm{k}_{0}^{2} \varepsilon_{\mathrm{m}}}}{\varepsilon_{\mathrm{m}} \sqrt{\beta^{2}-\mathrm{k}_{0}^{2} \varepsilon_{\mathrm{d}}}}=\tanh \left(\frac{L}{2} \sqrt{\beta^{2}-\mathrm{k}_{0}^{2} \varepsilon_{\mathrm{d}}}\right) .
$$

Fig. 2 shows the plasma frequency of $\mathrm{Ag}, \omega_{\mathrm{p}}$ (dark grey line), and $\mathrm{k}_{0}$ (navy line) plotted with the dispersion curves calculated from Eqs. (1)-(3) for the dispersion relation of a single Ag-air interface (green) and the two layers, MIM structures for $\mathrm{L}=40_{\text {(MIM) }}$ (teal), $\mathrm{L}=30_{\text {(MIM) }}$ (maroon), and

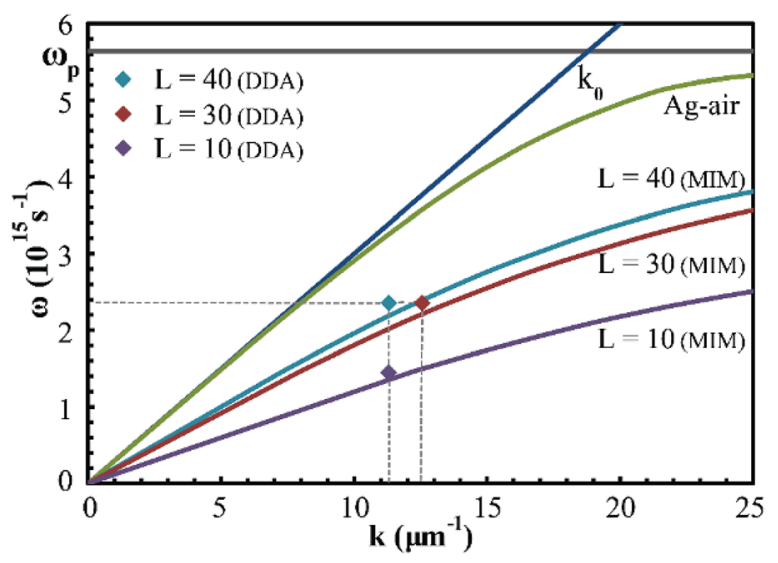

FIG. 2. Surface plasmon dispersion relation curves for a single Ag-air interface (green) and MIM structures with varied L (teal, maroon, indigo) calculated from Eqs. (1)-(3). Wavevector (diamond) for each of the periodic hole array structures from Figs. 1(b)-1(d) calculated using the DDA method are plotted with corresponding colors to the MIM counterparts. The free space wavevector, $\mathrm{k}_{0}$ (navy), and the plasma frequency of bulk $\mathrm{Ag}$ (dark grey) is also plotted for comparison. 
$\mathrm{L}=10$ (MIM) (indigo). All of the modes lie at higher wavevectors to the right of the light line, $\mathrm{k}_{0}$ and at lower frequencies than $\omega_{\mathrm{p}}$, indicating they are all propagating modes. When the second Ag layer is added, the dispersion curve shifts to the right and diverges at a lower frequency than the single interface. As L is decreased, the modes further shift to larger wavevectors and diverge at even lower frequencies due to the increase of $n_{\text {eff }}$ in the region. The shift of the dispersion curve in relation to $\mathrm{k}_{0}$ corresponds with the momentum mismatch between $\hbar k_{0}$ and $\hbar k_{\text {spp }}$.

For comparison, $\mathrm{k}_{\mathrm{spp}, \mathrm{DDA}}$ (diamonds) from the measured $\lambda_{\text {spp,DDA }}$ obtained from the $|\mathrm{E}|^{2}$ plots in Figs. 1(b)-1(d) are included at corresponding $\mathrm{L}_{(\mathrm{MIM})}$ distances. For the periodic hole array with $\lambda_{0}=800 \mathrm{~nm}$, when $\mathrm{L}=40 \mathrm{~nm}, \lambda_{\text {spp,MIM }}$ $=510 \mathrm{~nm}$ is red shifted to $\lambda_{\text {spp,DDA }}=556 \mathrm{~nm}$, and when $\mathrm{L}=30, \lambda_{\text {spp, } \mathrm{MIM}}=500 \mathrm{~nm}$ is red shifted to $\lambda_{\text {spp,DDA }}=556 \mathrm{~nm}$. For $\mathrm{L}=10 \mathrm{~nm}$ with $\lambda_{0}=1300 \mathrm{~nm}, \lambda_{\text {spp,MIM }}=463 \mathrm{~nm}$ is red shifted to $\lambda_{\mathrm{spp}, \mathrm{DDA}}=500 \mathrm{~nm}$, where the $\mathrm{k}_{\mathrm{spp}, \mathrm{DDA}}$ of the $\mathrm{L}=10 \mathrm{~nm}$ is the same as the $\mathrm{k}_{\mathrm{spp}, \mathrm{DDA}} \mathrm{L}=30 . \mathrm{k}_{\mathrm{spp}, \mathrm{DDA}}$ correlates with $\mathrm{k}_{\mathrm{spp}, \mathrm{MIM}}$ but is blue shifted to higher frequencies as a result of the coupling effects in the array. The addition of the periodic hole array results in a smaller momentum mismatch between $\hbar k_{\text {spp }}$ and $\hbar k_{0}$, shifting $k_{\text {spp }}$ closer to $k_{0}$. However, decreasing the film spacing allows for excited SPP modes on the silver interface to exist at optical frequencies and efficiently propagate light through the waveguide despite the reduced dimensions of the channel.

To test the limits of light confinement, we isolate the holes along the $\mathrm{Z}$ axis in $400 \mathrm{~nm}$ wide channels with a $\mathrm{Y}$ periodicity of $500 \mathrm{~nm}$, by including a $100 \mathrm{~nm}$ sidewall barrier between adjacent holes as shown in Fig. 3(a). The $|E|^{2}$ in the $\mathrm{YZ}$ plane for $\mathrm{L}=10 \mathrm{~nm}$ with $\mathrm{Z}$ polarized incident light with $\lambda_{0}=1020 \mathrm{~nm}$ is shown in Fig. 3(b), which demonstrates that light can propagate over a distance of $10 \mu \mathrm{m}$ when the channels are isolated with a dimension of $10 \times 400 \mathrm{~nm}^{2}$. The wavelength of the MIM structure calculated from Eq. (1), $\lambda_{\text {spp,MIM }}=389 \mathrm{~nm}$, red shifts with the addition of the channel walls to $\lambda_{\text {spp,DDA }}=500 \mathrm{~nm}$. To further confine the light, we reduce the channel width to equal that of the diameter of a hole, $d=300 \mathrm{~nm}$. At the same $\lambda_{0}$, when the channel width is reduced from $400 \mathrm{~nm}$ to $300 \mathrm{~nm}, \lambda_{\text {spp,DDA }}=500 \mathrm{~nm}$ red shifts to $\lambda_{\mathrm{spp}, \mathrm{DDA}}=556 \mathrm{~nm}$, achieving a smaller momentum
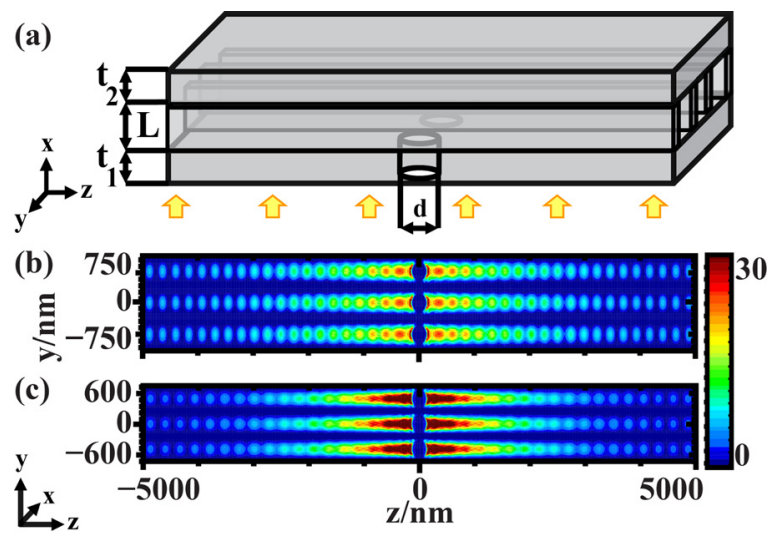

FIG. 3. (a) Schematic of an isolated hole array separated by $100 \mathrm{~nm}$ walls to form isolated channels along the $\mathrm{Z}$ axis. Electric field contour plots between the two layers in the $\mathrm{YZ}$ plane at a wavelength of $1020 \mathrm{~nm}$ for channel widths of (b) $400 \mathrm{~nm}$ and (c) $300 \mathrm{~nm}$.
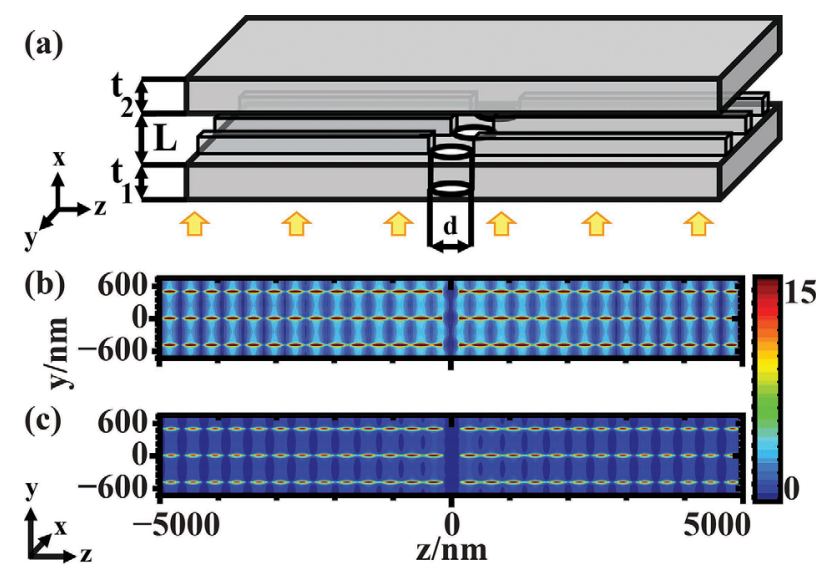

FIG. 4. (a) Schematic of an open hole array with ridges along the $\mathrm{Z}$ axis with $10 \mu \mathrm{m}$ periodic holes. Electric field contour plots between the two layers in the YZ plane of pseudo-coaxial structures with a spacing between the coaxial and top film of $10 \mathrm{~nm}$ and $\mathrm{L}=$ (b) $30 \mathrm{~nm}$ and (c) $40 \mathrm{~nm}$ at an incident wavelength of $1300 \mathrm{~nm}$.

mismatch between the corresponding wavevectors. The calculated $|\mathrm{E}|^{2}$ shown in Fig 3(c) reveals that light at a wavelength of $1020 \mathrm{~nm}$ can be trapped by a single hole and propagated along a $10 \mu \mathrm{m}$ long channel with cross sectional dimension of $10 \times 300 \mathrm{~nm}^{2}$.

To further test the limits of light confinement in the waveguide, we remove the channel walls and add a $20 \mathrm{~nm}$ wide ridge which extends from the center edges of adjacent holes every $10 \mu \mathrm{m}$ along the $\mathrm{Z}$ axis to form a new waveguide structure as shown in Fig. 4(a). The distance between the ridge and the top film layer is fixed at $10 \mathrm{~nm}$ while $\mathrm{L}$ is increased from $30 \mathrm{~nm}$ to $40 \mathrm{~nm}$. The function of the ridge is different than that of the walls, where the ridge serves to facilitate propagation within the highly confined ridge region compared to the inter film region, while the walls act as barriers to fully confine the holes along the $\mathrm{Z}$ axis.

Fig. 4(b) demonstrates that for $\lambda_{0}=1300 \mathrm{~nm}$ and $\mathrm{L}=30 \mathrm{~nm}$ with a $20 \mathrm{~nm}$ high ridge, light preferentially propagates along the extremely small region between the ridge and the $t_{2}$, rather than in the region between $t_{1}$ and $t_{2}$, with $\lambda_{\text {spp }}=740 \mathrm{~nm}$. Amazingly, when $\mathrm{L}$ is increased to $40 \mathrm{~nm}$ with a ridge height of $30 \mathrm{~nm}$, the electric field intensity decreases even further in the interfilm region while leaving the ridge region intensity nearly unchanged with a red shifted $\lambda_{\text {spp }}=769 \mathrm{~nm}$, as shown in Fig. 4(c). The wavelength of the SPPs propagating at the interface of the top metal film is shown to be determined mainly by the spacing between the two films and not the spacing between the ridge and top film. Consequently, as the interfilm spacing is increased and $\mathrm{n}_{\mathrm{eff}}$ is decreased, the effective wavelength of the SPP modes is red shifted, but light confinement and propagation is preserved along the ridge.

We numerically demonstrated that light can be trapped, confined, and propagated between two silver films at an extremely subwavelength dimension of $10 \times 20 \mathrm{~nm}^{2}$. The wavelength of the SPP modes is dependent on $n_{d, \text { eff }}$ of the dielectric region in the waveguide, which is dictated by the distance between films. The most efficient waveguides have a smaller spacing between films due to the increased coupling of SPP modes at the Ag-air interfaces so that light preferentially 
propagates in a $10 \mathrm{~nm}$ region, despite the drastic mismatch between $\lambda_{0}$ and the waveguide dimensions. The SPP wavelength can be tuned by changing the distance between films with the propagation preservation achieved by including a ridge to confine light. The extreme two-dimensional confinement and propagation of light by the two-layer film can provide useful information for the future design of waveguides at extreme subwavelength dimensions.

We are thankful for the support of this research by the National Science Foundation and Office of Naval Research.

${ }^{1}$ S. E. Thompson and S. Parthasarathy, Mater. Today 9(6), 20-25 (2006). ${ }^{2}$ W. Cai, W. Shin, S. Fan, and M. L. Brongersma, Adv. Mater. 22(45), 5120-5124 (2010).

${ }^{3}$ J. Chen, Z. Li, S. Yue, and Q. Gong, Appl. Phys. Lett. 97(4), 041113 (2010).
${ }^{4}$ J. A. Dionne, H. J. Lezec, and H. A. Atwater, Nano Lett. 6(9), 1928-1932 (2006).

${ }^{5}$ Y. Fang, Z. Li, Y. Huang, S. Zhang, P. Nordlander, N. J. Halas, and H. Xu, Nano Lett. 10(5), 1950-1954 (2010).

${ }^{6}$ E.-S. Kwak, J. Henzie, S.-H. Chang, S. K. Gray, G. C. Schatz, and T. W. Odom, Nano Lett. 5(10), 1963-1967 (2005).

${ }^{7}$ C. Ropers, C. C. Neacsu, T. Elsaesser, M. Albrecht, M. B. Raschke, and C. Lienau, Nano Lett. 7(9), 2784-2788 (2007).

${ }^{8}$ S. Zhu, T. Y. Liow, G. Q. Lo, and D. L. Kwong, Opt. Express 19(9), 8888-8902 (2011).

${ }^{9}$ F. Yu, H. Wang, and S. Zou, J. Phys. Chem. C 114(5), 2066-2069 (2010).

${ }^{10}$ H. Wang and S. Zou, Appl. Phys. Lett. 94(7), 073119 (2009).

${ }^{11}$ E. M. Purcell and C. R. Pennypacker, Astrophys. J. 186, 705-714 (1973).

${ }^{12}$ B. T. Draine and P. J. Flatau, J. Opt. Soc. Am. A 11(4), 1491-1499 (1994).

${ }^{13}$ E. D. Palik, Handbook of Optical Constants of Solids (Academic Press, Boston, 1998).

${ }^{14}$ B. T. Draine and P. J. Flatau, J. Opt. Soc. Am. A 25(11), 2693-2703 (2008).

${ }^{15}$ R. Gordon and A. Brolo, Opt. Express 13, 1933-1938 (2005). 